\title{
e-Phaïstos
}

e-Phaïstos

Revue d'histoire des techniques / Journal of the history

of technology

V-2 | 2016

Histoire des techniques en Afrique de l'Ouest

\section{Production de verre à boire et phénomènes de mode en région parisienne à l'époque moderne}

Production of Glass to Drink and Fashion Phenomena in the Paris Region in Modern Times.

\section{Saubade Roussel}

\section{(2) OpenEdition}

\section{Journals}

Édition électronique

URL : http://journals.openedition.org/ephaistos/5838

DOI : 10.4000/ephaistos.5838

ISSN : 2552-0741

Éditeur

IHMC - Institut d'histoire moderne et contemporaine (UMR 8066)

Édition imprimée

Date de publication : 15 juin 2016

Pagination : 80-90

ISSN : 2262-7340

Référence électronique

Saubade Roussel, «Production de verre à boire et phénomènes de mode en région parisienne à l'époque moderne », e-Phaïstos [En ligne], V-2 | 2016, mis en ligne le 15 mars 2020, consulté le 19 juin 2020. URL : http://journals.openedition.org/ephaistos/5838 ; DOI : https://doi.org/10.4000/ephaistos 5838 


\title{
Production de verre à boire et phénomène de mode en région parisienne à l'époque moderne
}

\author{
Saubade Roussel \\ Masterante en Histoire des Techniques \\ Université Paris 1 Panthéon-Sorbonne
}

\section{Introduction}

Depuis le XIX ${ }^{\mathrm{e}}$ siècle, l'historiographie a insisté sur l'influence des productions vénitiennes sur la verrerie européenne à partir de la fin du XVe siècle. Résultant d'abord d'un goût de l'aristocratie pour la verrerie de Venise, qui s'est spécialisée dans les productions de luxe, cette influence a perduré tout au long des XVI e et XVII e siècles, qui ont vu apparaître dans toute l'Europe des verreries qualifiées dès l'origine de «à la façon de Venise ». Celles-ci, imitant les productions vénitiennes, étaient fabriquées d'une part par des verriers italiens émigrés, d'autre part par une main-d'œuvre plus locale reprenant les formes et les techniques italiennes, dans un contexte de fort brassage des techniques, dont les modalités restent complexes à étudier ${ }^{1}$.

Toutefois, ces «façons de Venise » se sont insérées dans un système de production préexistant, afin de compléter l'offre à destination d'un marché local en forte expansion. Plus précisément, les débuts d'une massification de la consommation ont entraîné, en réaction, une demande pour des produits permettant de se démarquer dans la sphère sociale par l'affichage d'une certaine ostentation. Ainsi, à partir du XVIe et à plus forte raison dans la première moitié du XVIIe siècle, les verres de goût italien ont été employés dans ce but ${ }^{2}$; les verriers implantés en Ile-deFrance, d'abord italiens ayant amené leurs produits et matières premières, puis locaux ayant acquis à leur tour le savoir-faire nécessaire, ont donc approvisionné le marché francilien en objets dont le style a évolué en fonction de modes en vigueur dans la haute société.

Il semble donc essentiel d'appréhender le lien entre le marché soumis à des phénomènes de mode, et le contexte voire le complexe technique de production, si l'on veut pouvoir expliquer les évolutions de celui-ci au cours de l'époque moderne. Cependant, en l'absence de sources en provenance directe des ateliers de fabrication pouvant nous renseigner sur le fonctionnement de ceux-ci, il nous faut faire appel à une documentation périphérique pour cerner ces éléments. La vaisselle en verre elle-même, et en particulier ses caractéristiques stylistiques et techniques, connue à travers les exemplaires de musées et les découvertes archéologiques, et mise en parallèle avec les connaissances sur les ateliers franciliens, constitue de fait le principal vecteur d'information sur l'expression matérielle de ces phénomènes de mode et les résultats des complexes techniques en évolution. Après avoir détaillée celleci, nous nous pencherons sur les transferts techniques impliqués par cette appropriation progressive de formes italiennes par des verriers français, ainsi que sur les pratiques d'acquisition sur le marché parisien : en particulier, se pose la question d'une réciprocité plus ou moins forte de l'influence du marché et de la volonté des 
consommateurs sur les producteurs, les phénomènes de mode étant par nature cycliques et à double sens (la demande conditionne l'offre, mais le renouvellement de l'offre peut également créer la demande).

\section{Mode du verre à l'époque moderne}

\section{L'existence des modes}

L'un des marqueurs de la modernité du point de vue de la « culture matérielle », ou pourrait-on dire de l'expression matérielle de la culture, est le développement globalement conjoint (même si sur des temporalités et avec des modalités quelque peu différentes) à la fois d'une consommation de masse, impliquant une production de biens également plus importante, et d'une "culture des apparences »3. Ceci a entraîné la mise en place progressive, très embryonnaire au XVIe et tout à fait affirmée au XVIII siècle, de phénomènes d'émulation sociale consistant à afficher des objets à la fois nouveaux et au moins en apparence luxueux, ceux-ci tombant en désuétude du point de vue de leur fonction sociale (même si encore parfaitement intacts quant à leur fonction utilitaire) une fois leur possession généralisée. Ces phénomènes de «mode», bien connus dans le domaine du vêtement4, sont également tout à fait présents dans les autres aspects de la culture matérielle (et même de la culture en général)5. De plus, à l'intérieur de ces mouvements généraux pour un type d'objet, des variations potentiellement rapides constituent en elles-mêmes des courants de mode sur un cycle plus court: il peut en particulier s'agir des types de décors sur un objet comme la céramique fine, régulièrement renouvelés même si la technique de production restait généralement la même.

\section{La mode du verre}

Si la faïence a marqué le XVIII ${ }^{\text {e }}$ siècle, le verre a lui été l'un des éléments constitutifs de la culture des apparences du XVIIe siècle, voire depuis la fin du XVIe siècle. Cette fois, le référent original n'est pas une production extra-européenne mais les verres de grande qualité produits dans les ateliers de Venise depuis la fin du Moyen Âge. En effet, des innovations notables effectuées dans cette ville à la fin du XVe siècle quant à la matière et au faciès de la verrerie, avec en particulier l'apparition du " cristallo », un verre incolore d'une transparence exceptionnelle, ont amené la clientèle européenne fortunée européenne et notamment française à s'intéresser au verre à des fins d'ostentation et à passer commande de verres vénitiens. Un phénomène de diffusion de la stylistique et de la technique a ensuite eu lieu aux $\mathrm{XVI}^{\mathrm{e}}$ et $\mathrm{XVII}{ }^{\mathrm{e}}$ siècles, avec l'installation de verriers italiens dans des ateliers disséminés en Europe, puis le transfert de leurs techniques aux artisans locaux. Ceci a eu pour conséquence une remarquable homogénéité de la verrerie, ce qui rend difficile l'attribution de la verrerie muséale à une origine précise7. Cette tendance apparaît aussi au sein du mobilier archéologique $^{8}$ et le même problème d'identification et d'attribution se pose pour la verrerie parisienne, comme l'a relevé Jorge Barrera lors de l'étude du corpus archéologique parisien de référence de la Cour Napoléon du Louvre9.

C'est seulement depuis une dizaine d'années que les travaux d'histoire de l'art et d'archéologie commencent à permettre de résoudre ces problèmes, d'identifier les différences au sein de cette ressemblance et d'esquisser des typologies régionales ${ }^{10}$. Toutefois, bien que des styles locaux différents apparaissent, il n'est cependant pas toujours possible de rattacher à chaque style un centre de production ${ }^{11}$ : le verre faisait l'objet d'un commerce international et les ateliers adaptaient leur production à la demande des différents marchés ${ }^{12}$, si bien que les artisans eux-mêmes pouvaient se trouver incapable de faire la différence entre les productions locales et les verreries d'importation produites pour le même marché ${ }^{13}$. Cependant, il a existé une émancipation stylistique 
discrète mais bien réelle, influencée par de nouveaux centres de production de luxe, comme Nevers ou Anvers. Ce constat montre que le système de production s'adaptait pour répondre à une demande bien précise. Le même phénomène s'observe en Angleterre, où les lettres du marchand John Greene à son fournisseur de verres vénitien Allesio Morelli présentent de nombreux dessins représentant le profil des objets qu'il souhaitait importer ${ }^{14}$.

\section{Les productions}

A l'aune de la documentation archivistique actuelle, il n'est pas possible de présenter d'aperçu approfondi du marché de la verrerie francilienne au XVII ${ }^{2}$ siècle $^{15}$. Il est toutefois envisageable que certaines sources, notamment notariales (contrats de ventes, inventaires après décès) nous permettent à l'avenir d'avancer des éléments supplémentaires, comme cela a été le cas pour les Pays-Bas ${ }^{16}$ ou en Belgique ${ }^{17}$. Pour la fin du XVIII ${ }^{2}$ siècle notamment, une série de livres de comptes de marchands verriers conservés aux Archives municipales et départementales de Paris ${ }^{18}$ nous renseigne ainsi de manière très précise sur les quantités, natures et prix des produits vendus, voire sur les pratiques d'achat. On peut y déceler une très grande diversité de modes de consommation, du tenancier de taverne achetant plusieurs centaines de verres en une fois au particulier n'en acquérant qu'un seul.

Ces modes de consommation sont à mettre en relation avec l'existence de différentes gammes de production répondant à une logique d'émulation et de consommation de masse liée aux phénomènes de mode ${ }^{19}$. En effet, les fouilles archéologiques ont fourni des quantités importantes ${ }^{20}$ de verreries de toutes qualités s'apparentant stylistiquement à celles de très bonne qualité conservées actuellement dans les musées. Parallèlement à une production commune d'une stylistique différente, ces objets, désignés par Corine Maitte comme "demi-luxe» ou «luxe bas de gamme » ${ }^{21}$, semblent résulter de phénomènes d'imitation des exemplaires d'ostentation; ils ont fait leur apparition dans la seconde moitie du XVIe siècle et se sont atténués dans la seconde moitié du XVIIe siècle, où il devient difficile de distinguer une production de «luxe», le verre devenant un produit de consommation de masse sortant du champ de l'ostentation ${ }^{22}$.

\section{Fabrication des verres}

\section{L'implantation des ateliers}

La première production attestée de verres d'inspiration italienne en Île-de-France est celle du bolonais d'origine altaraise Teseo Mutio, qui a installé un atelier à Saint-Germain-en-Laye en 1551, à la demande de Henri $\mathrm{II}^{23}$. Son privilège, unique dans le Royaume, est l'un des premiers documents attestant de cette production dans la région, puisqu'il emploie directement le terme de «façon de Venise » 24 : le but affiché est clairement d'imiter la production vénitienne ${ }^{25}$. A la fin du XVIe siècle, après une tentative avortée à Melun, l'altarais Sarrode a établi un nouvel atelier aux portes de Paris, à Saint-Germain-des-Prés, puis au tournant du siècle, Jean Maréchal, français pour sa part, s'est installé au même lieu, Sarrode ayant perdu la faveur royale pour avoir refusé de former des apprentis français ${ }^{26}$. Maréchal obtint en 1606 le privilège exclusif de produire et vendre la verrerie à Paris et à 30 lieues alentour, cet état de fait se poursuivant jusqu'aux années $1640^{27}$, et ce n'est qu'en 1677 que les marchands verriers et faïenciers parisiens eurent l'autorisation de s'approvisionner à plus de 20 lieues autour de la ville ${ }^{28}$. Bien que ce monopole théorique n'ait certainement pas été entièrement respecté dans la pratique (des verres de Venise, voire des contrefaçons, étant importées depuis des régions périphériques avant cette date ${ }^{29}$ ), il indique au moins une volonté du pouvoir de conserver une production locale, ou du moins proche du centre de consommation parisien. 
Malheureusement, bien que le four de l'un des ateliers de Saint-Germain-des-Prés ait très probablement été retrouvé archéologiquement ${ }^{30}$, aucune documentation complète ou suffisamment précise ne nous permet de préciser le fonctionnement interne de ces fabriques. Par ailleurs, en l'absence de plus d'indications permettant de mettre en lien productions et centres de production, et notamment de signatures de verriers $3^{1}$, la source la plus indiquée pour suivre l'évolution des productions répondant à la demande de la clientèle reste les verres eux-mêmes, et tout particulièrement ceux découverts dans les fouilles archéologiques.

\section{Les verres eux-mêmes, d'un point de vue technique}

D'un point de vue technique, l'historiographie distingue trois grandes catégories de verres à boire aux XVIe et XVIIe siècles : les gobelets, qui reposent directement sur le fond du récipient; les verres à pied ou à jambe en une seule paraison façonnés selon la technique du refoulement ${ }^{32}$, et les verres à jambe en plusieurs paraisons, façonnés par apports successifs de verre. L'étude de chacun de ces soustypes d'objet présente des problématiques propres.

Le gobelet est une forme née au XVe siècle et restée courante dans les corpus archéologiques jusqu'au milieu du XVIe siècle, mais semble se raréfier par la suite. Parfois orné de décors moulés ou rapportés, sa forme générale reste simple et ce n'est pas le type d'objet le plus marqué par les évolutions techniques et stylistiques des $\mathrm{XVI}{ }^{\mathrm{e}}$ et XVII siècles.

Les verres en une seule paraison comprennent principalement les verres à pied refoulé33, qui présentent un pied creux obtenu en repoussant le fond du contenant vers l'intérieur du verre ; selon leur forme ils sont qualifiés de bitronconiques ou de biconiques. Ces objets, que l'on trouve couramment jusqu'à la fin du XVI ${ }^{\mathrm{e}}$ siècle, sont principalement présents dans les données archéologiques à l'état fragmentaire, le pied étant la partie la plus fréquemment conservée, ce qui pose des problèmes d'identification. En effet, ces pieds refoulés ne sont pas caractéristiques de la verrerie à boire et sont également présents sur d'autres objets comme des carafes ou des coupes sur pied. Le faible potentiel informatif des données disponibles rend donc l'étude de l'évolution de ces objets difficile.

Enfin, les verres en plusieurs paraisons présentent à l'époque moderne des formes très diverses. Ils sont caractérisés par la présence d'une jambe souvent elle-même formée de sous-éléments qui présentent une très grande diversité technique et stylistique. Cette catégorie couvre une large gamme d'objets allant de formes relativement simples 34 à des objets très ornementés comme les verres à serpentine ou à mufles de lion 35 .

Les caractéristiques stylistiques et techniques de ces objets peuvent bien renseigner sur l'évolution des modes et de la production, mais leur grande diversité peut poser problème pour leur description et leur comparaison. A l'image du travail de Jorge Barrera sur la verrerie de la Cour Napoléon du Louvre, une approche typo-chronologique s'avère nécessaire, afin de rassembler les verres présentant des similitudes morphologiques et techniques en des types, dont il est possible d'étudier l'évolution et la distribution à travers les collections muséales et archéologiques. Cette méthode présente toutefois des limites, les objets se ne se distinguant que par des différences subtiles qui varient de façon continue, rendant difficile de délimiter des types distincts permettant de rendre compte de façon exhaustive des caractéristiques des pièces ${ }^{36}$.

Le classement typologique demande donc à être complété par une description approfondie des caractéristiques techniques et morphologique des pièces, qui doit, pour constituer une base de travail pertinente, faire appel à un vocabulaire précis et univoque. L'élaboration d'un thésaurus et le choix de termes pertinents au sein du vocabulaire employé dans la documentation historique et archéologique existante constitue donc une 
première étape indispensable à l'étude37. Ce vocabulaire descriptif permet notamment de faire directement référence aux éléments témoins de processus techniques particuliers : traces de pontil, soudures ou amolisses marquant l'apport d'une nouvelle paraison, pied ourlé caractéristique d'une forme refoulée, décors obtenus par divers procédés techniques (moulage, travail à l'outil, etc.), lèvre de contenant rebrûlée au feu, etc.

Afin de proposer une solution pratique pour la description intersites de ces éléments et leur comparaison, nous avons ainsi mis au point une base de données spécifique permettant de rendre compte des spécificités de ce type d'artefact 38 . Celle-ci inclut la possibilité de subdiviser chaque partie de l'objet en autant de sousensembles que nécessaires, permettant grâce à cette structure en arborescence de décrire dans le détail chaque objet, serait-il doté de caractéristiques tout à fait uniques impossibles à prévoir dans un système informatif « fixé ». Cet outil, dans lequel nous avons inclus un certain nombre de corpus de référence pour l'Île-de-France moderne39, nous a ainsi permis de déterminer les grandes lignes de l'évolution de la typologie des verres de luxe et semi-luxe sur cette période, afin de la mettre en relation d'une part avec l'évolution des modes, et d'autre part avec les changements intervenus dans le complexe technique de production.

\section{1}
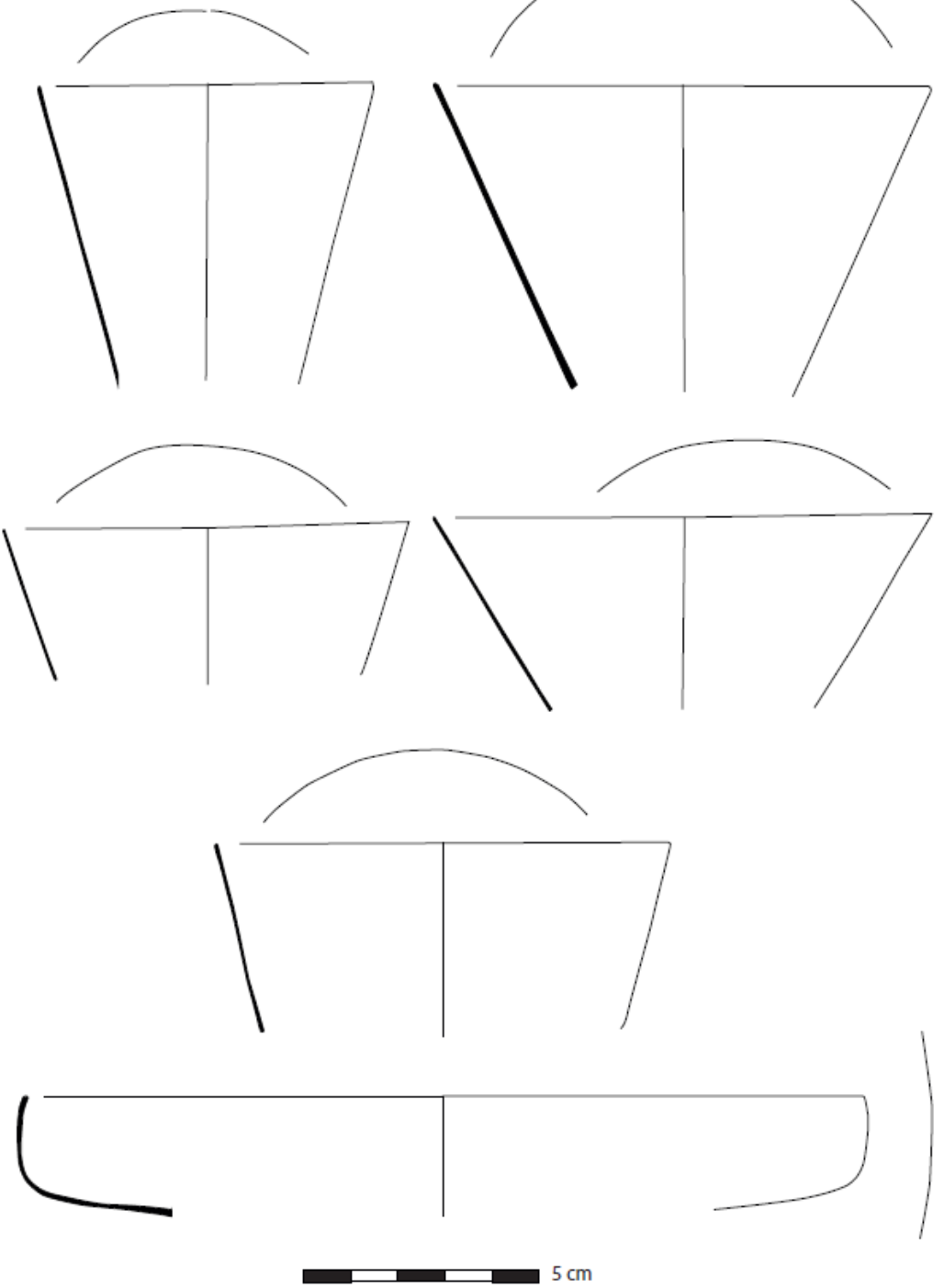

Figure 1 : Représentation en coupe de quelques contenants de verres à boire à jambe de tailles variées, issus d'un contexte de latrine moderne (US 1831) du prieuré de Villiers-le-Bel. La dernière figure pourrait correspondre à une coupe à fruits. 
L'évolution générale témoigne ainsi d'une homogénéisation marquée des productions au cours de la période temporelle considérée. En effet, on note jusqu'au début du XVII siècle une grande variété dans les gammes de production, allant d'une verrerie commune de facture très médiocre (avec une matière de mauvaise qualité, colorée involontairement, au pied désaxé, etc...) à des objets très fins (comme des coupes sur jambe). On relève également la présence de pièces simples mais d'une facture soignée, en particulier des verres à jambe refoulée40, dont la pâte n'est pas exempte d'impuretés mais qui ont été volontairement colorés en bleu pour masquer celles-ci. Cette utilisation des couleurs est bien marquée dans la première moitié du XVII ${ }^{e}$ siècle, des corpus comme celui de l'îlot Cygne à Saint-Denis paraissant notoirement hétéroclites de ce point de vue.

$\mathrm{Au}$ contraire, la facture s'est clairement régularisée dans la seconde moitié du XVIIe siècle, y compris pour des formes considérées comme « communes » (verres à pied, coupelles). Celle-ci est nettement plus régulière, avec une certaine maturité du processus technique, notamment au niveau des profils des pieds. De même, la couleur est beaucoup moins employée, la grande majorité des pièces étant incolores. Toutefois, si les pieds des pièces ont eu clairement tendance à être homogénéisés, ce choix n'a pas du tout été fait quant aux récipients, qui sont restés de formes et de contenances très différentes (cf. fig.1). Il s'agit donc manifestement d'un choix délibéré au niveau stylistique, seuls certains éléments étant homogénéisés alors qu'aucune limitation technique n'aurait empêché de faire de même sur les autres.

Nous retrouvons ainsi bien ici la relation entre les phénomènes de mode et le complexe technique de production. En effet, les modes ont manifestement façonné le verre «idéal » attendu par le marché, avec une production attendue laissant moins de place aux imperfections techniques, même camouflées sous la couleur (voire au contraire mettant en valeur la pureté de la pâte par le caractère incolore), et requérant une homogénéisation au moins de certaines parties du verre, comme les pieds, mais pas de toutes (soit une possibilité de garder des caractéristiques ostentatoires tout en entrant dans un vocabulaire commun du verre de table). Or, toutes ces modifications ont une origine technique dans le domaine du verre: la perfection de la pâte, la standardisation des formes, ou encore l'application - ou non - de couleur dépendent toutes directement du processus de fabrication ${ }^{41}$. Cette évolution des goûts a donc supposé un phénomène de dialogue entre les centres de production et les consommateurs ${ }^{42}$, d'alignement des différents centres sur les mêmes techniques, ou encore d'une homogénéisation de la qualité et de la nature des matières premières utilisées.

Ce lien entre la technique et la culture des apparences de l'époque moderne, particulièrement important dans le domaine du verre, mais loin d'y être limité, ouvre donc une porte sur une approche systémique large du fonctionnement de ce marché francilien, dans lequel on devine une interconnexion poussée entre les différents acteurs à une échelle locale, régionale voire extra-régionale. Cet exemple montre également la nécessité d'inclure l'étude archéologique dans le travail sur ces thématiques impliquant des objets physiques, une approche encore peu développée pour l'époque moderne mais qui montre ici son potentiel, considérable aussi bien du point de vue d'approches traditionnellement historiques qu'archéologiques. Il conviendra à l'avenir de se pencher plus avant sur la mise en place, l'organisation et le fonctionnement des réseaux techniques mis en évidence dans le présent article, recherche faisant actuellement l'objet de notre mémoire de Master II.

1 Voir par exemple : MAITTE Corine, Les chemins de verre. Les migrations des verriers d'Altare et de Venise, XVIe-XIXe siècles, Rennes, Presses Universitaires de Rennes, 2009. BRAUNSTEIN 
Philippe, «Innovations italiennes et répercussions en Europe : Introduction ", Les innovations verrières et leur devenir : actes du deuxième colloque international de l'association Verre et Histoire, Nancy, 2009.

2 Dans la seconde moitié du siècle, la documentation notamment archéologique montre une diminution de cette caractéristique, à mettre probablement en relation avec le développement du miroir qui prend à son tour ce rôle. DE ROCHEBRUNE MarieLaure, "La céramique et le verre dans la peinture française au temps de Louis XIII », Dossiers de l'art, n86, 2002, p. 30-41.

3 ROCHE Daniel, La culture des apparences : Une histoire du vêtement (XVIIe-XVIIIe siècle), Paris, Fayard, 1989.

4 CARDON Dominique, Mémoires de teinture. Voyage dans le temps chez un maître des couleurs, Paris, CNRS Éditions, 2013. FIGEAC, Michel (dir.), L'ancienne France au quotidien, Paris, Armand Colin, 2014, p. 337-341.

5 On peut citer en exemple la porcelaine chinoise Ming, objet de luxe et d'ostentation réservé aux élites sociales pendant la plus grande partie de l'époque moderne; au XVIIIe siècle, une série d'innovations techniques dans les manufactures européennes permirent de produire en quantités importantes des faïences de bonne qualité pouvant rivaliser en apparence avec les porcelaines originales, permettant ainsi une diffusion de cette mode dans la société, si bien que le XIXe siècle a vu ce type d'objet, à présent très répandu, perdre son prestige social.

6 TAIT Hugh, Five Thousand Years of Glass, Londres, British Museum Press, 1991, p. 159-160. Les plus anciennes commandes françaises de verres vénitiens connues sont quatre pièces aux armes d'Anne de Bretagne, datées de son règne (1499-1514) (voir DE ROCHEBRUNE Marie-Laure, «Le verre de Venise et "à la façon de Venise » en France aux XVIe et XVIIe siècles », Revue de la Société des Amis du Musée National de Céramique, Sèvres, no13, 2004, p. 17.

7 BAUMGARTNER Erwin, Venise et façon de Venise. Verres renaissance du Musée des arts décoratifs, Paris, Union centrale des arts décoratifs, 2003.

8 «Des pièces découvertes en France pourraient se confondre avec le mobilier exhumé en Italie » (FOY Danièle, préface dans CABART Hubert, La verrerie archéologique : Dieulouard et l'Est de la France aux XVIe et XVIIe siècles, Nancy, Presses Universitaires de Nancy, 2011, p.9.)

9 «il devient complexe de conclure sur l'origine des verres archéologiques, d'autant plus que l'on constate [...] des objets très semblables dans des régions très éloignées " (BARRERA Jorge, «L'influence italienne de la verrerie sur la moitié nord de la France », in MENDERA Marja (dir.), Archeologia e storia della produzione del vetro preindustriale, Borgo San Lorenzo, All'insegna Del Giglio, 1991, p. 345-357.).
10 Par exemple, un groupe de verres du musée des Arts Décoratifs de Bordeaux présentant une stylistique particulière, attribués par la tradition à une production du Sud-Ouest, a pu voir cette origine confirmée par la comparaison avec des objets retrouvés dans des fouilles d'ateliers (HÉBRARD-SALIVAS Catherine, «Qu'en est-il de l'appellation « attribution verre du Sud-Ouest »? ", in OLIVIE Jean-Luc et RUBINI Constance, Verres d'usage et d'apparat de la Renaissance au XIXe siècle. La collection du Mesnil au musée des Arts décoratifs, hôtel de Lalande, Bordeaux, Paris, Norma, 2014, p. 135.).

11 DE ROCHEBRUNE Marie-Laure, «Venetian and Façon de Venise glass in France in the 16th and 17th centuries », in PAGE Jutta-Annette (éd.), Beyond Venice. Glass in Venetian style, 1500-1750, Corning, Corning Museum of Glass, 2004, p. 150.

LHERMITE-KING Sylvie, Cent verres français 1550-1750. Trésors des collections privées. Catalogue d'exposition du 13 au 26 octobre 2008, Paris, À la façon de Venise, 2008, p. 7.

12 MAITTE Corine, Les chemins de verre. Les migrations des verriers d'Altare et de Venise, XVIe-XIXe siècles, Rennes, Presses Universitaires de Rennes, 2009, p. 82-83.

13 BAUMGARTNER Erwin, Venise et façon de Venise. Verres renaissance du Musée des arts décoratifs, Paris, Union centrale des arts décoratifs, 2003, p. 24.

14 WILLMOTT Hugh, "Venetian and Facon de Venise glass in England », in PAGE Jutta-Annette (éd.), Beyond Venice. Glass in Venetian style, 1500-1750, Corning, Corning Museum of Glass, 2004, p. 296-297.

15 «L'analyse systématique, à la fois synchronique et diachronique, des prix des verres récoltés au fil des archives, n'a pas encore été réalisée. " (MAITTE Corine, Les chemins de verre. Les migrations des verriers d'Altare et de Venise, XVIeXIXe siècles, Rennes, Presses Universitaires de Rennes, 2009, p. 85).

16 LIEFKES Reino, « Façon de Venise glass in the Netherlands », in PAGE Jutta-Annette (éd.), Beyond Venice. Glass in Venetian style, 1500-1750, Corning, Corning Museum of Glass, 2004, p. 236.

17 DE STAELEN Carolien et CALUWÉ Danielle, «A cupboard and a basket with some glasses... a glass without a foot ", Annales du XVIIe Congrès de l'Association Internationale pour l'Histoire du verre, Anvers, 2006, p. 317-367.

18 Archives départementales de Paris, série D5B6. Celle-ci regroupe les derniers livres de compte des marchands parisiens en faillite dans les décennies précédent la Révolution, lesquels étaient conservés par le Châtelet de Paris. Parmi les centaines de cotes de cette série, celles qui concernent des marchands verriers (tous ou presque également faïenciers) sont les 214, 856, 1850, 2113, 2212, 2621, 2622, 3365, 3382, 3392, 4045, 4113, 4533, $4905,5883,5884,5885,5886,5887,5888$ et 5889 . 
19 MAITTE Corine, Les chemins de verre. Les migrations des verriers d'Altare et de Venise, XVIe-XIXe siècles, Rennes, Presses Universitaires de Rennes, 2009, p.88.

20 La fouille de la Cour Napoléon du Louvre a ainsi livré plus de 1200 objets en verre creux issus de la période moderne (BARRERA Jorge, "Le verre à boire des fouilles de la Cour Napoléon du Louvre (Paris) ", Annales du XIe Congrès de l'Association Internationale pour l'Histoire $d u$ verre, Amsterdam, 1990, p. 359), et plusieurs sites de l'est de la France ont également livré des corpus de plusieurs centaines d'individus (337 à Dieulouard, 444 à Montbéliard, 804 à Epinal, etc. ; voir CABART Hubert, La verrerie archéologique : Dieulouard et l'Est de la France aux XVIe et XVIIe siècles, Nancy, Presses Universitaires de Nancy, 2011, p. 41).

21 MAITTE Corine, Les chemins de verre. Les migrations des verriers d'Altare et de Venise, XVIe-XIXe siècles, Rennes, Presses Universitaires de Rennes, 2009, p. 79, 83

22 DE ROCHEBRUNE Marie-Laure, « La céramique et le verre dans la peinture française au temps de Louis XIII », Dossiers de l'art, $\mathrm{n}^{\circ} 86,2002$, p. 30-41.

23 DE ROCHEBRUNE Marie-Laure, «Venetian and Façon de Venise glass in France in the 16th and 17th centuries ", in PAGE Jutta-Annette (éd.), Beyond Venice. Glass in Venetian style, 1500-1750, Corning, Corning Museum of Glass, 2004. VANRIEST Elise, «La «Verrerie de Paris » à Saint-Germaindes-Prés (1597-1610) », Bulletin de l'AFAV, 2015, p. 97.

24 PHILIPPE Michel, Naissance de la verrerie moderne, XIIeXVIe siècle. Aspects économiques, techniques et humains, Turnhout, Brepols, 1998, p. 39.

25 Arch. nat. Xia 8618, fol. 51, 13 juin 1551, autorisation donnée à Theseo Mutio pour la fabrication de verre cristallin, cité dans PHILIPPE Michel, Naissance de la verrerie moderne, XIIe-XVIe siècle. Aspects économiques, techniques et humains, Turnhout, Brepols, 1998, p. 39.

26 VANRIEST Elise, « La « Verrerie de Paris » à Saint-Germaindes-Prés (1597-1610) », Bulletin de l'AFAV, 2015, p. 97.

27 VANRIEST Elise, « La « Verrerie de Paris » à Saint-Germaindes-Prés (1597-1610) », Bulletin de l'AFAV, 2015, p. 97.

28 Arrêt du Parlement du 21 juillet 1677, cité dans DE LESPINASSE René, Les corporations et métiers de la ville de Paris. .2: Orfèvrerie, sculpture, mercerie, ouvriers en métaux, bâtiment et ameublement, Paris, Imprimerie nationale, 1892, p.761.

29 Bibl. Nat. Fr. 21733, cité dans PHILIPPE Michel, Naissance de la verrerie moderne, XIIe-XVIe siècle. Aspects économiques, techniques et humains, Turnhout, Brepols, 1998, p. 41.

30 VANRIEST Elise, « La « Verrerie de Paris » à Saint-Germaindes-Prés (1597-1610) », Bulletin de l'AFAV, 2015, p. 97-100.
PETIT Michel et SERVAT Edmond, «Un quartier privilégié du Paris antique : le Sénat », Dossiers d'Archéologie, $\mathrm{n}^{\circ} 7,1974$, p. 41-47.

31 MAITTE Corine, "Imitation, copie, contrefaçon, faux : définitions et pratiques sous l'Ancien Régime », Entreprises et Histoire, I, $\mathrm{n}^{\mathrm{o}} 78,2015$, p. 35 .

32 Une paraison correspond à une masse de verre en fusion cueillie à la canne et ajoutée à l'objet. Le refoulement consiste à utiliser un outil pour repousser le fond de la boule soufflée vers l'intérieur, de facon à former une base ou un pied (voir ROUSSEL Saubade, Étude de deux corpus de verre moderne en Pays de France : fouilles du 4, rue du Cygne à Saint-Denis (93) et du Prieuré de Villiers-le-Bel (95), mémoire de M2 Recherche, Université Paris 1 Panthéon-Sorbonne, 2015, p. 98).

33 Également qualifiés de «verre sur piédouche » ou parfois de " gobelet sur piédouche ».

34 Comme les verres à jambe pleine du type 15 de la typologie de la Cour Napoléon du Louvre.

35 Respectivement les types 17 et 16 de la même typologie.

36 ROUSSEL Saubade, Étude de deux corpus de verre moderne en Pays de France : fouilles du 4, rue du Cygne à Saint-Denis (93) et du Prieuré de Villiers-le-Bel (95), mémoire de M2 Recherche, Université Paris 1 Panthéon-Sorbonne, 2015, p.: 5354 .

37 Un tel vocabulaire normalisé a été proposé dans des travaux antérieurs (ROUSSEL Saubade, Étude de deux corpus de verre moderne en Pays de France: fouilles du 4, rue du Cygne à Saint-Denis (93) et du Prieuré de Villiers-le-Bel (95), mémoire de M2 Recherche, Université Paris 1 Panthéon-Sorbonne, 2015, p. 63-71) ; il s'agit d'une première proposition ne prétendant pas à l'exhaustivité.

38 ROUSSEL Saubade, Étude de deux corpus de verre moderne en Pays de France : fouilles du 4, rue du Cygne à Saint-Denis (93) et du Prieuré de Villiers-le-Bel (95), mémoire de $\mathrm{M} 2$ Recherche, Université Paris 1 Panthéon-Sorbonne, 2015, p. 72 75 .

39 Ces corpus sont issus des fouilles du 4, rue du Cygne à SaintDenis (GORET, Jean-François, Saint-Denis (Seine-Saint-Denis). 4, rue du Cygne. Fouille programmée. Rapport triennal ; campagnes 201O, 2011 et 2012, Saint-Denis, Unité d'Archéologie de Saint-Denis, 2013.) et du prieuré de Villiers-le-Bel (GENTILI François (dir.), Villiers-le-bel, 1 rue Gambeta et 2 bis rue Victor Gouffé. Au coeur du village : cimetière, habitat seigneurial et prieuré médiévaux et modernes. Rapport de fouille préventive. Inrap centre Ile-de-France, 2014.)

40 Type 12 de la typologie de référence de la Cour Napoléon du Louvre. 
41 A la différence par exemple d'une céramique dans laquelle le décor exact appliqué n'est pas directement lié à une technique spécifique.

42 Sachant que les propriétaires de verreries pouvaient euxmêmes fréquenter la bonne société parisienne, simplifiant ainsi ce processus. 


\section{Bibliographie}

BARRERA Jorge, « Le verre à boire des fouilles de la Cour Napoléon du Louvre (Paris) », Annales du XIe Congrès de l'Association Internationale pour l'Histoire du verre, Amsterdam, 1990, p. 347364.

BARRERA Jorge, "L'influence italienne de la verrerie sur la moitié nord de la France», in MENDERA Marja (dir.), Archeologia e storia della produzione del vetro preindustriale, Borgo San Lorenzo, All'insegna Del Giglio, 1991, p. 345-357.

BAUMGARTNER Erwin, Venise et façon de Venise. Verres renaissance du Musée des arts décoratifs, Paris, Union centrale des arts décoratifs, 2003.

BRAUNSTEIN Philippe, «Innovations italiennes et répercussions en Europe : Introduction ", Les innovations verrières et leur devenir : actes du deuxième colloque international de l'association Verre et Histoire, Nancy, 2009.

CARDON Dominique, Mémoires de teinture. Voyage dans le temps chez un maître des couleurs, Paris, CNRS Éditions, 2013.

FIGEAC, Michel (dir.), L'ancienne France au quotidien, Paris, Armand Colin, 2014.

GENTILI François (dir.), Villiers-le-bel, 1 rue Gambeta et 2 bis rue Victor Gouffé. Au cœur du village : cimetière, habitat seigneurial et prieuré médiévaux et modernes. Rapport de fouille préventive. Inrap centre Ile-de-France, 2014.

GORET, Jean-François, Saint-Denis (SeineSaint-Denis). 4, rue du Cygne. Fouille programmée. Rapport triennal ; campagnes 2010, 2011 et 2012, Saint-Denis, Unité d'Archéologie de Saint-Denis, 2013.

HÉBRARD-SALIVAS Catherine, «Qu'en est-il de l'appellation "attribution verre du SudOuest »? », in OLIVIE Jean-Luc et RUBINI
Constance, Verres d'usage et d'apparat de la Renaissance au XIXe siècle. La collection du Mesnil au musée des Arts décoratifs, hôtel de Lalande, Bordeaux, Paris, Norma, 2014, p. 134-137.

DE LESPINASSE René, Les corporations et métiers de la ville de Paris. T.2: Orfêvrerie, sculpture, mercerie, ouvriers en métaux, bâtiment et ameublement, Paris, Imprimerie nationale, 1892.

LHERMITE-KING Sylvie, Cent verres français 1550-1750. Trésors des collections privées. Catalogue d'exposition du 13 au 26 octobre 20o8, Paris, A la façon de Venise, 2008.

LIEFKES Reino, "Facon de Venise glass in the Netherlands », in PAGE Jutta-Annette (éd.), Beyond Venice. Glass in Venetian style, 1500-1750, Corning, Corning Museum of Glass, 2004, p. 226269.

MAITTE Corine, «Imitation, copie, contrefaçon, faux: définitions et pratiques sous l'Ancien Régime », Entreprises et Histoire, I, n ${ }^{\circ} 78,2015$.

MAITTE Corine, Les chemins de verre. Les migrations des verriers d'Altare et de Venise, $X V I^{e_{-}}$ $X I X^{e}$ siècles, Rennes, Presses Universitaires de Rennes, 2009.

PETIT Michel et SERVAT Edmond, «Un quartier privilégié du Paris antique: le Sénat», Dossiers d'Archéologie, ${ }^{\circ} 7,1974$, p. 41-47.

PHILIPPE Michel, Naissance de la verrerie moderne, XII ${ }^{e}-X V I^{e}$ siècle. Aspects économiques, techniques et humains, Turnhout, Brepols, 1998.

ROCHE Daniel, La culture des apparences: Une histoire du vêtement (XVIIe-XVIIIe siècle), Paris, Fayard, 1989.

ROCHE, Daniel, Histoire des choses banales. Naissance de la consommation, XVII ${ }^{e}-X I X^{e}$ siècle, Paris, Fayard, 1997.

DE ROCHEBRUNE Marie-Laure, «La céramique et le verre dans la peinture française au 
temps de Louis XIII », Dossiers de l'art, n86, 2002, p. 30-41.

DE ROCHEBRUNE Marie-Laure, "Le verre de Venise et "à la façon de Venise » en France aux $\mathrm{XVI}$ et XVII ${ }^{\mathrm{e}}$ siècles ", Revue de la société des amis du musée national de la céramique, $\mathrm{n}^{\circ} 13,2004, \mathrm{p}$. 17-32.

ROUSSEL Saubade, Étude de deux corpus de verre moderne en Pays de France : fouilles du 4, rue du Cygne à Saint-Denis (93) et du Prieuré de Villiers-le-Bel (95), mémoire de M2 Recherche, Université Paris 1 Panthéon-Sorbonne, 2015.

DE STAELEN Carolien et CALUWÉ Danielle, "A cupboard and a basket with some glasses... a glass without a foot ", Annales du XVII e Congrès de l'Association Internationale pour l'Histoire $d u$ verre, Anvers, 2006, p. 370-376.

TAIT Hugh, Five thousand years of glass, Londres, British Museum Press, 1991.

VANRIEST Elise, "La « Verrerie de Paris » à Saint-Germain-des-Prés (1597-1610) », Bulletin de l'AFAV, 2015, p. 97-100.

WILLMOTT Hugh, "Venetian and Facon de Venise glass in England », in PAGE Jutta-Annette (éd.), Beyond Venice. Glass in Venetian style, 1500175o, Corning, Corning Museum of Glass, 2004, p. 270-307. 